\title{
Miniaturization of MEMS Capacitive Accelerometers Fabricated Using Silicon-on-insulator-MEMS Technology
}

\author{
Jiannan Liu* and Hideo Muro \\ Chiba Institute of Technology, 2-17-1 Tsudanuma, Narashino, Chiba 275-0016, Japan \\ (Received July 31, 2017; accepted November 16, 2017)
}

Keywords: miniaturization, capacitive, beam configuration, sensitivity, resonant frequency

In our previous work, we studied capacitive micro-accelerometers fabricated using a simple silicon-on-insulator (SOI)-MEMS technology and the feasibility of an accelerometer with a beam length as long as the side length of the proof mass. In this work, we investigate the dependences of their sensitivities and resonant frequencies on the beam parameters and its configuration and study the method of designing the stuctures of capactive accelerometers with beam lengths as long as the side length of the proof mass in order to miniaturize the device.

\section{Introduction}

Since MEMS sensors were initially commercialized as accelerometers for airbag systems in the 1990s, they have been widely used for various applications such as movement detection in hand-held mobile terminals and control devices, ${ }^{(1)}$ and the technologies have made significant progress in performance, compactness, and reliability. The common detection mechanisms for accelerometers are piezoelectric, ${ }^{(2)}$ piezorsistive, ${ }^{(3,4)}$ and capacitive. ${ }^{(5)}$

The two main fabrication technologies are bulk micromachining ${ }^{(6)}$ and surface micromachining. ${ }^{(7)}$ Because of several advantages, that is, low manufacturing cost, high machining precision, and easy integration with microelectronics, surface micromachining has become the mainstream in recent years. Surface micromachining sensors have small initial capacitance and proof mass since they are constructed from membranes. Factors influencing microstructures with respect to sensitivity are not limited to proof mass but also the length, width, and structural shape of the spring beam. In general, since the displacement or sensitivity is proportional to the cube of the beam length, increasing the beam length is more advantageous with respect to to the sensitivity than a folded beam structure. ${ }^{(8-13)}$ However, owing to various constraints, folded-beam structures have been widely used in accelerometer design. For example, in the design of the mask, various parts should be densely arranged in a small area and folded-beam structures are easy to pack in a limited area. Design optimization and systematic analysis have not yet been extensively carried out. On the other hand, MEMS sensor and actuator devices are modeled by simple equations and their downscaling effects on their performance, such as sensitivity and resonant frequency, have been investigated. ${ }^{(14)}$

*Corresponding author: e-mail: ryukennan304@gmail.com http://dx.doi.org/10.18494/SAM.2018.1716 
In the models, the effects of electric field increment due to the size reduction effect were taken into account, but there was no study on keeping the performance such as sensitivity and unchanged while reducing the proof mass size. In our previous work, ${ }^{(15)}$ we studied capacitive micro-accelerometers fabricated using a simple silicon-on-insulator (SOI)-MEMS technology, by which comparatively thick planar structures required for high-performance sensors can be realized by deep reactive ion etching (DRIE) and subsequent oxide etching, investigated the dependence of their sensitivity and resonant frequency on beam parameters and its configuration, and studied the feasibility of an accelerometer with a beam length as long as the side length of the proof mass. The resonant frequencies were intensively investigated since comparably low resonant frequencies of the higher order modes could cause incorrect output in some applications.

This paper focuses on capacitive micro-accelerometers fabricated using a simple SOI-MEMS and various types of such accelerometers are investigated to clarify the design technology for miniaturizing the device.

\section{Device Structures}

\subsection{Test samples}

Basic structures of test devices are shown in Fig. 1. The design includes three basic structures of both-sides-supported accelerometers. All have the same beam width of $3 \mu \mathrm{m}$ and similar comb electrode structures. The proof masses are (a) A-Type (which is the reference in our previous paper): $1000 \times 1000 \mu \mathrm{m}^{2}$, (b) B-Type: $750 \times 750 \mu \mathrm{m}^{2}$, and (c) C-Type: $500 \times 500$ $\mu \mathrm{m}^{2}$. The aim of studying the $\mathrm{B}$ and $\mathrm{C}$ types is to determine the feasibility of an accelerometer with a folded-beam length as long as the side length of the proof mass when its proof mass is miniaturized compared with the reference Type-A.

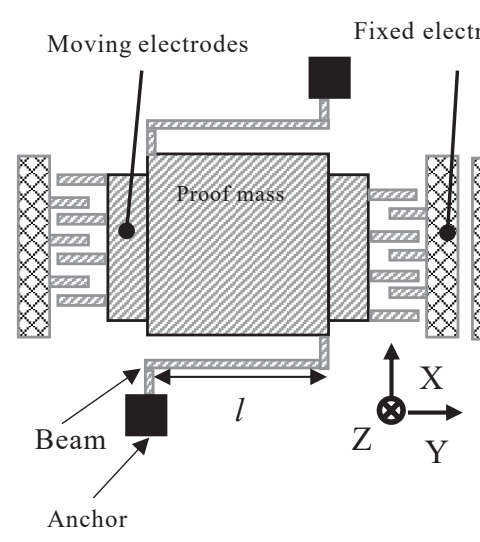

(a)

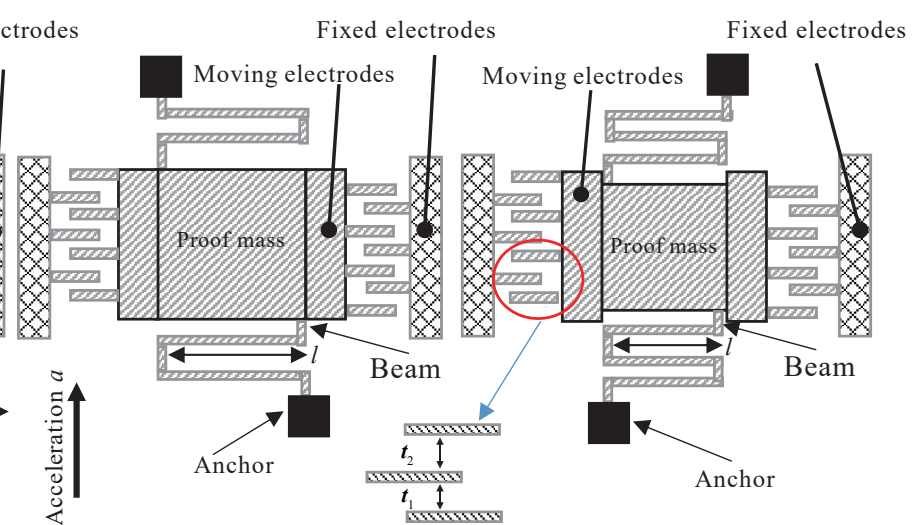

(b)

(c)

Fig. 1. (Color online) Basic structures of capacitive accelerometers. (a) A-type, (b) B-type, and (c) C-type. 
The beam length of each basic structure is as follows. (a) The entire beam length $L$ is as long as the side length of the proof mass, namely, $1000 \mu \mathrm{m}$. (b) The entire beam length $L$ is $l$ $\times n$, where $l$ is the beam length that is as long as the side length of the proof mass, namely, 750 $\mu \mathrm{m}$, and the beam is connected to the proof mass with a structure folded $n$ times. (c) The entire beam length $L$ is $l \times n$, where $l$ is the beam length that is as long as the side length of the proof mass, namely, $500 \mu \mathrm{m}$, and the beam is connected to the proof mass with a structure folded $n$ times.

The prototypes were fabricated using a standard process in the MEMS foundry. Figure 2 shows the cross-sectional view of the accelerometers, where the movable portion is released by etching the buried oxide film. The Si (device layer) thickness is $25 \mu \mathrm{m}$, the oxide thickness is $2 \mu \mathrm{m}$, the handle wafer (substrate) thickness is $400 \mu \mathrm{m}$, and the Au thickness is $500 \mathrm{~nm}$. The structure is manufactured by a trench-etching process on the SOI substrate, as shown in Fig. 3, where the process begins with $150 \mathrm{~mm}$ n-type double-sides-polished SOI wafers.

\subsection{Principle of operation}

The principle of operation of the both-sides-supported accelerometers is explained in the following. The displacement of the proof mass can be written as

$$
\Delta x=\frac{m a l^{3}}{2 E b d^{3}},
$$

where $m$ is the proof mass, $a$ is the applied acceleration, $l$ is the length, $b$ is the width, $d$ is the thickness of the beam, and $E$ is Young's modulus. The spring constant $k$ of a both-sidessupported beam can be written as

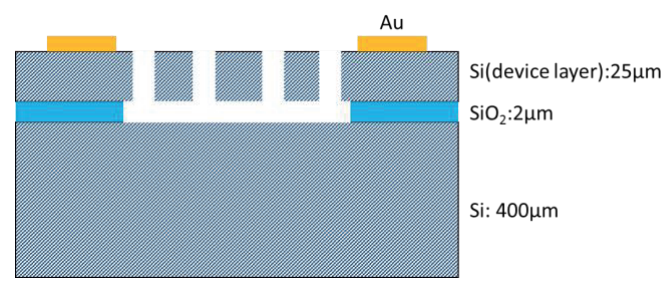

Fig. 2. (Color online) Cross-sectional view of the accelerometer.

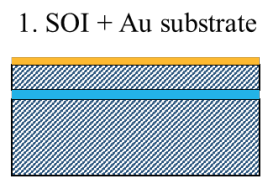

2. Resist(PR) coating \&
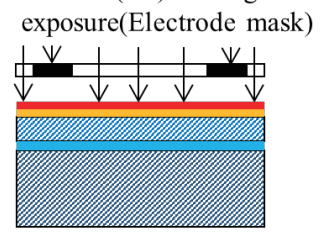

4. Resist(PR) coating \&

exposure (Electrode mask)

$\vee \vee \vee|\vee| \vee|\vee| \vee \vee V$

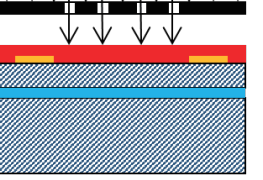

5. Si etch + remove resist

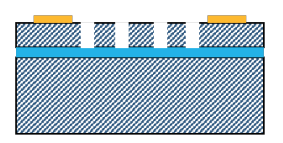

3. Au etch + remove the resist

6. $\mathrm{SiO}_{2}$ etch (release etch)
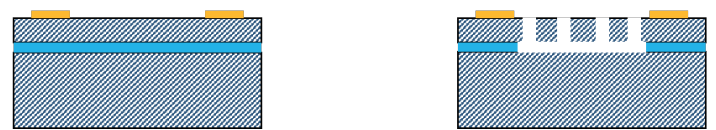

Fig. 3. (Color online) Fabrication process flow of SOI-MEMS accelerometer. 


$$
f=\frac{1}{2 \pi} \sqrt{\frac{2 E b d^{3}}{m l^{3}}} .
$$

The dependence of the resonant frequency on the beam parameters is expressed by

$$
\begin{gathered}
k=\frac{2 d^{3} b E}{l^{3}}, \\
\left(\frac{L}{n}\right)^{3} \times n=\frac{L^{3}}{n^{2}} .
\end{gathered}
$$

When the beam length is as long as the side length of the proof mass, the displacement $\Delta x$ is propotional to the cube of $l$. However, in the case of folded-beam accelerometers, the displacement is propotional to Eq. (4), where $n$ is the number of beam folds. Therefore, the sensitivity of the folded-beam type is higher than that of the nonfolded type by $L^{5} / n^{4}$. When the total beam length $L$ is fixed, and the side length of the proof mass is increased to $\eta l$, the sensitivity of the accelerometer with the increased proof mass is $\eta^{5}$ times that of the original sensitivity.

\section{Simulations}

Initially, the maximum displacement under $1 g$ acceleration was analyzed using the finite element method (FEM) simulator "IntelliSuite". Figure 4 shows the result. The abscissa is the entire length, and the ordinate is displacement $\Delta x$. The displacement of the A-Type is proportional to the cube of beam length, while those of the B-Type and C-Type are proportional to the entire beam length $L$ and enlarged by the fold number $n$. Compared with the C-Type sensor with folding, when the entire length of the A-Type with a nonfolded beam is the same as that of the C-Type, the calculated displacement $\Delta x$ is 14 times that of the C-Type. On the

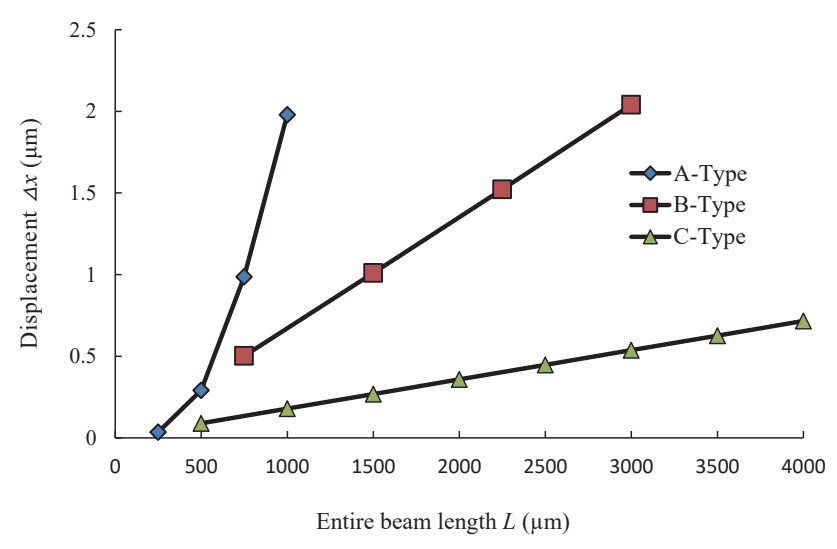

Fig. 4. (Color online) Calculated displacement vs entire beam length. 
other hand, the individual beam of the B-Type is $3 / 2$ times longer than that of the C-Type, and the calculated displacement $\Delta x$ is 5 times larger. The results of the simulation agree reasonably well with estimates obtained using the simple model.

Figure 5 shows the schematic diagrams of accelerometer resonant modes. Figure 5(a) shows the principal mode in which the proof mass moves along the $y$ axis. Figure 5(b) shows the second mode, where the proof mass twists around the $y$ axis. Figure 5(c) shows the third mode, in which the proof mass moves in the $z$ axis direction.

Figure 6 shows the calculated resonant frequencies, which depend on the entire beam length. The abscissa is for the entire beam length, and the ordinate is for the resonant frequency. The resonant frequency of the first mode $f_{1}$ is inversely proportional to the square root of the fifth power of the entire beam length $L$. The values of simulated results agree well with the theoretical values of the simple model.

Figure 7 shows the calculated results of the ratio $f_{2} / f_{1}$, where $f_{2}$ is the resonant frequency of the second mode. The calculated ratio is proportional to the entire beam length $L$ for the A-Type. In contrast, the calculated ratios are not dependent on the entire beam length $L$ for the B-Type and C-Type, where the ratios of the B-Type and C-Type are 6 and 5, respectively. These results seem to be related to the structures of the B-Type and C-Type, which incorporate folded beams and are more immune to torsion than the A-type which has no folded beams.

Figure 8 shows the calculated results of the ratio of resonant frequencies $f_{3} / f_{1}$, where $f_{3}$ is the resonant frequency of the third mode. The ratios of the B-Type decrease gradually with increasing entire beam length $L$, while the C-Type is not dependent on the entire beam length $L$. In contrast, the ratios of the A-Type increase from 7 to 8 as the entire beam length $L$ is increased. The spring constant in the $z$ axis direction for the C-Type decreases gradually as the entire beam length $L$ is increased. This seems to be caused by several connecting joints included in the beam, which could facilitate the motion in the $z$ axis direction by torsional deformation.

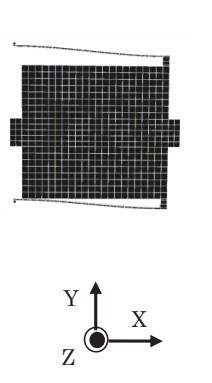

(a)

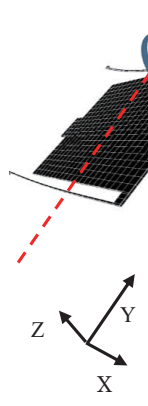

(b)

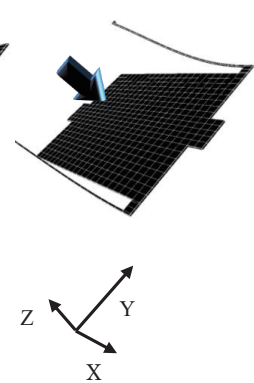

(c)

Fig. 5. (Color online) Shapes of the three resonant modes. (a) Mode 1, (b) Mode 2, and (c) Mode 3.

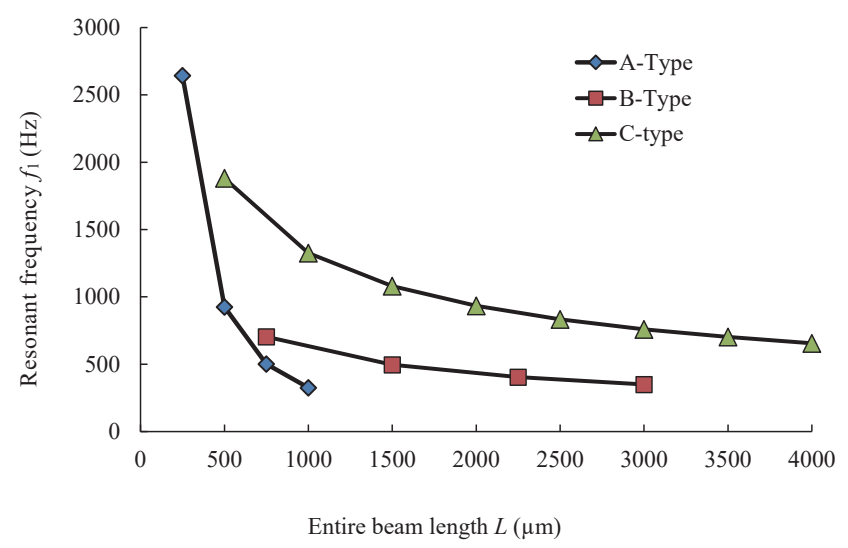

Fig. 6. (Color online) Calculated resonant frequencies of Mode 1. 


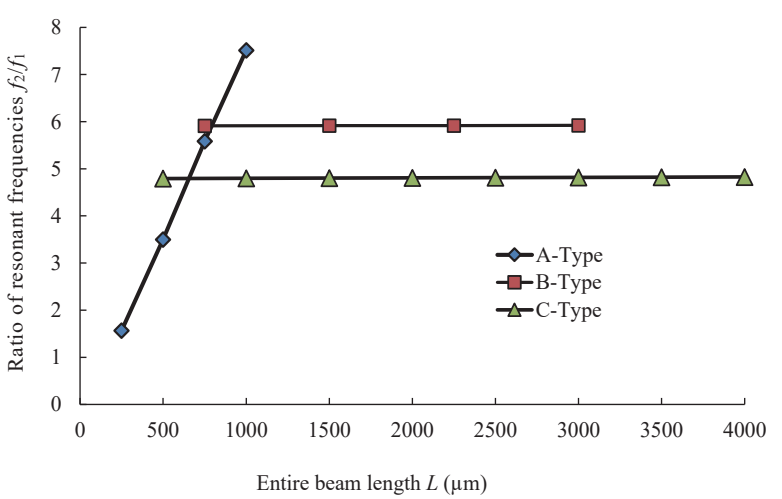

Fig. 7. (Color online) Calculated ratio of resonant frequencies Mode 2/Mode 1.

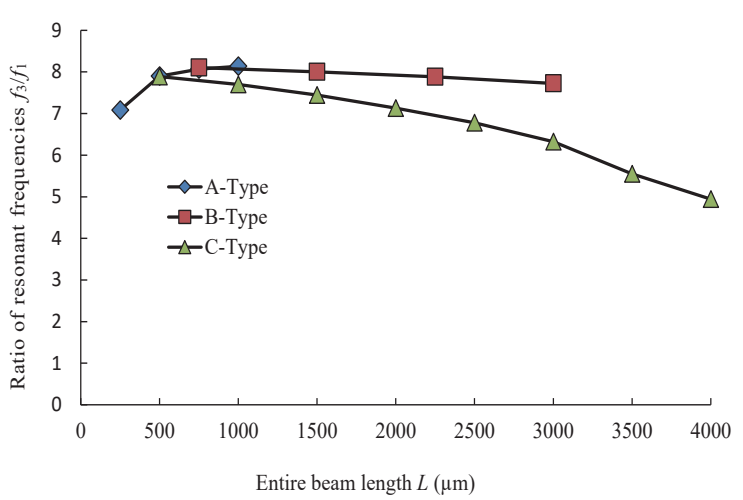

Fig. 8. (Color online) Calculated ratio of resonant frequencies Mode 3/Mode 1.

From these simulated results for resonant frequencies, it is noted that the resonant frequencies of higher orders could be larger than the principal resonant frequency by more than four times for the B-Type and C-Type, while the beam length should be longer than $750 \mu \mathrm{m}$ for the A-Type. Since the resonant frequencies of higher orders are sufficiently high, there might be little possibility of incorrect output.

\section{Experiments}

The three types of accelerometers shown in Fig. 1 were designed, and their prototypes were fabricated by SOI- MEMS technology. The details of dimensions and design parameters are listed in Table 1. Figures 9(a)-9(c) show the SEM images of these prototypes. The sensors were mounted in DIP28 ceramic packages, and aluminum wires were bonded.

The signal-processing circuit for sensor evaluation was designed as shown in Fig. 10. The output voltage of the amplifier was rectified using a diode and a capacitor and measured. The sensitivity and the resonant frequency were measured using this circuit. The AC signal was inverted by a phase-inverting circuit and input to one side of the sensing electrode. The output signal of the charge amplifier was amplified by the subsequent amplifier circuit. The output signal was rectified by the full wave rectification circuit. The sensitivity was evaluated using a rotary stage with the acceleration of gravity of $\pm 1 g$. Figure 11 shows the measured output voltages under $1--1 g$ acceleration. The measured result for the A-Type was $485 \mathrm{mV} / \mathrm{G}$, that of the B-Type with a beam folded 3 times was $463 \mathrm{mV} / \mathrm{G}$, and that of the C-Type with a beam folded 7 times was $251 \mathrm{mV} / \mathrm{G}$. These sensitivity values agree well with the theoretical values of the simple models.

Figure 12 shows the measured frequency responses obtained using a shaker. The shaker was 512-D made by EMIC, whose maximum displacement was $\pm 7 \mathrm{~mm}$. The frequency was swept from $50 \mathrm{~Hz}$ to $1 \mathrm{kHz}$ under $1 \mathrm{~g}\left(9.8 \mathrm{~m} / \mathrm{s}^{2}\right)$. The measurement was carried out two times and the basic frequency step was $10 \mathrm{~Hz}$. The small resonant peak of each type could be observed. The resonant frequency of the A-Type was $340 \mathrm{~Hz}$ with an output voltage of $467 \mathrm{mV}$, that of the 
Table 1

Parameters of prototype sensors.

\begin{tabular}{lccc}
\hline Model & A-type & B-type & C-type \\
\hline Proof mass $m(\mathrm{~kg})$ & $5.1216 \mathrm{E}-08$ & $4.09728 \mathrm{E}-08$ & $3.17539 \mathrm{E}-08$ \\
Whole beam length $L(\mu \mathrm{m})$ & 1000 & 2250 & 4000 \\
Spring $k(\mathrm{~N} / \mathrm{m})$ & 0.03 & 0.04 & 0.14 \\
Displacement $\triangle x(\mu \mathrm{m} / \mathrm{G})$ & 0.75 & 1.66 & 1.66 \\
\cline { 2 - 4 } Gap $t(\mu \mathrm{m})$ & \multicolumn{3}{c}{$t_{1}=2, t_{2}=5$} \\
Young's modulus $E(\mathrm{GPa})$ & \multicolumn{3}{c}{2328} \\
\cline { 2 - 4 } Density $\left(\mathrm{kg} / \mathrm{m}^{3}\right)$ & 375 & 349 & 701 \\
Resonant frequency $f(\mathrm{~Hz})$ & 512.5 & 505.7 & 304.4 \\
Calculated sensitivity $(\mathrm{mV} / \mathrm{G})$ & \multicolumn{3}{c}{}
\end{tabular}

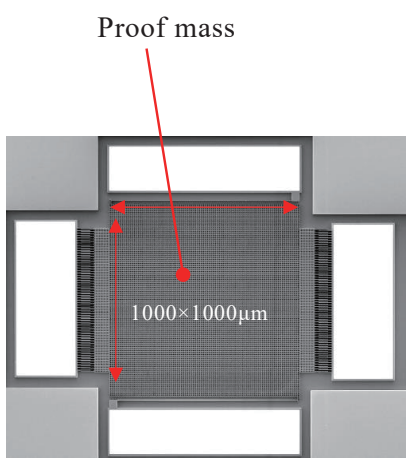

(a)

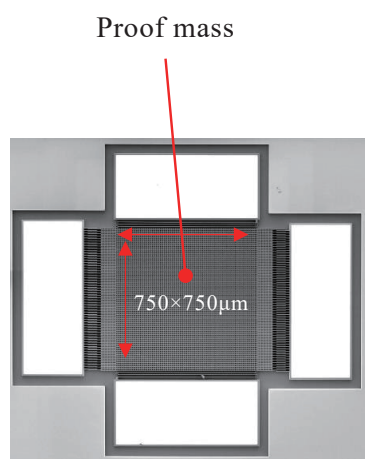

(b)

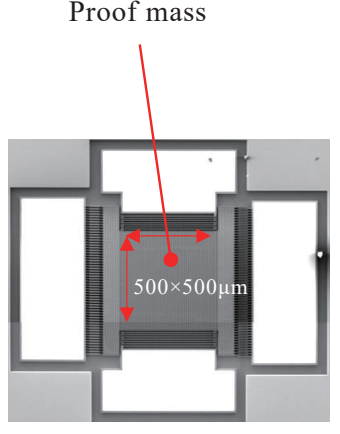

(c)

Fig. 9. (Color online) SEM images of the fabricated prototypes. (a) A-Type, (b) B-Type, and (c) C-Type.

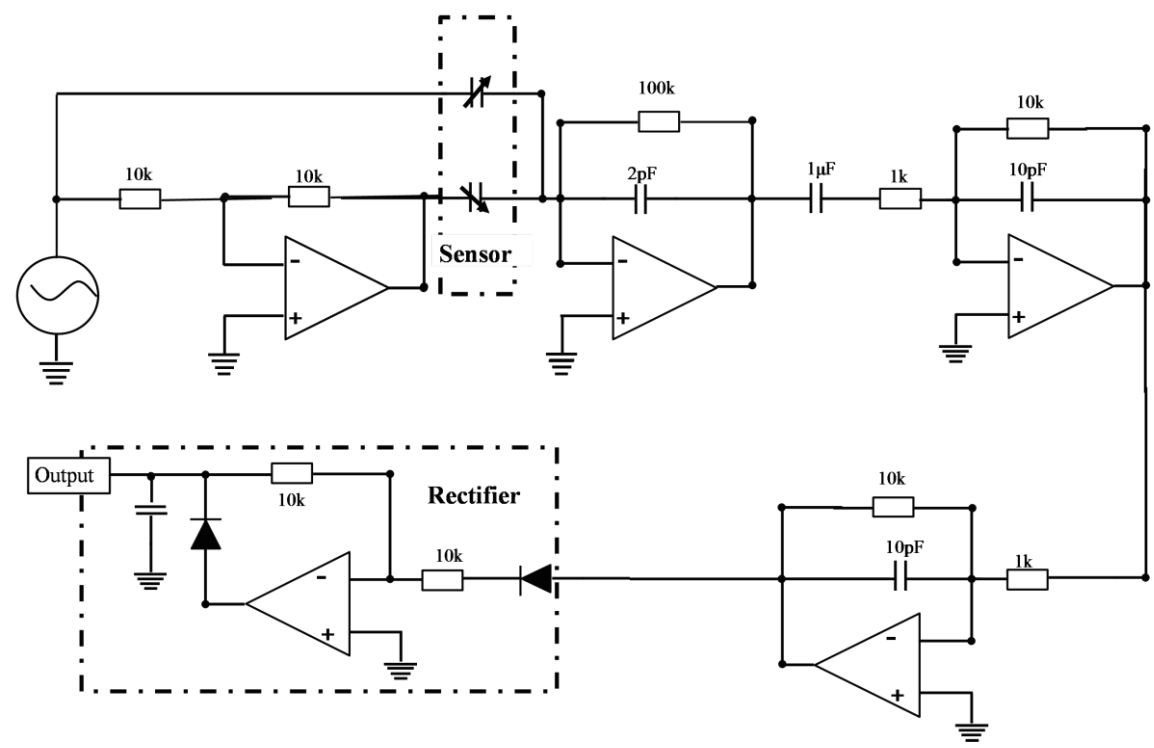

Fig. 10. Circuit diagram of signal-processing circuitry. 


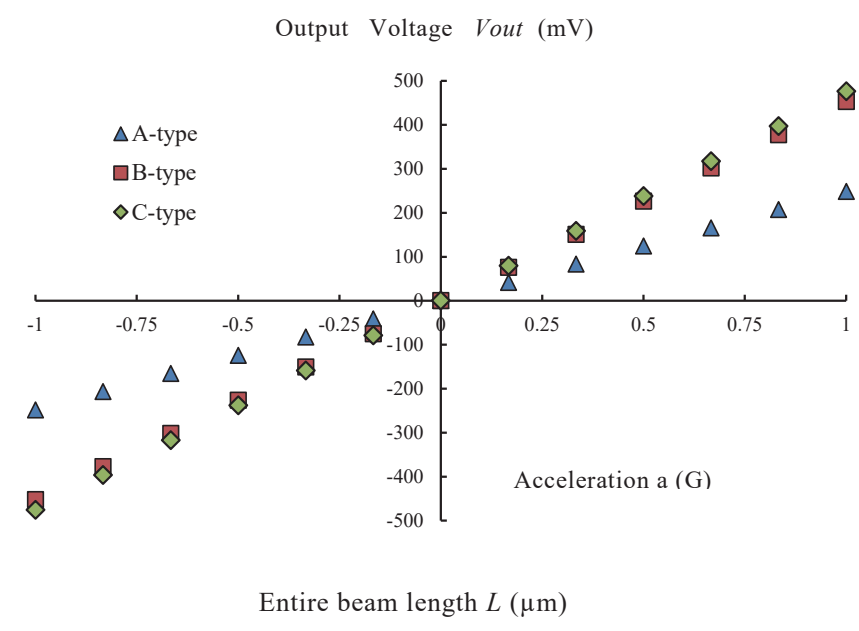

Fig. 11. (Color online) Measured dependences of the output voltage on the applied acceleration.

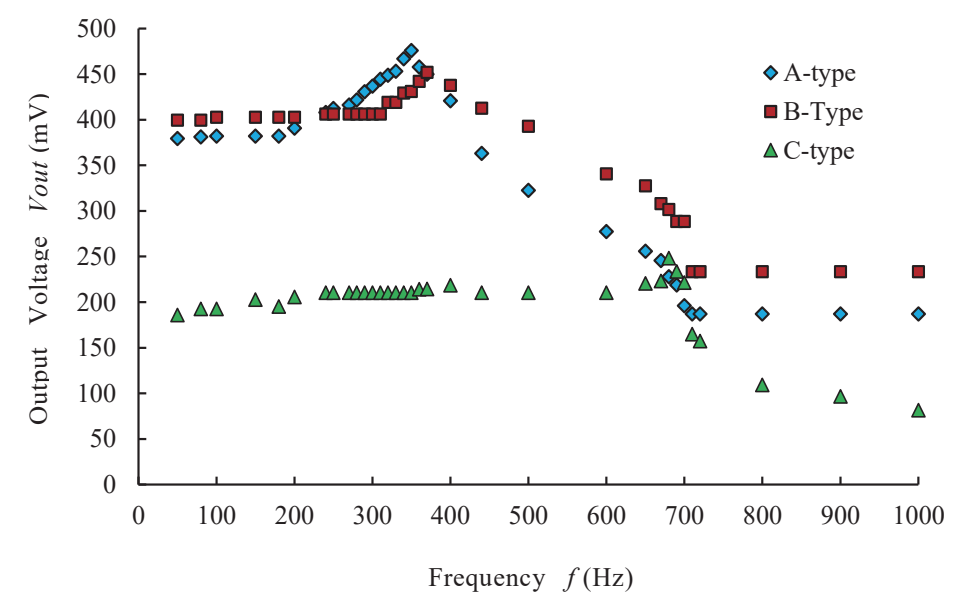

Fig. 12. (Color online) Measured frequency characteristics.

B-Type was $370 \mathrm{~Hz}$ with an output voltage of $452 \mathrm{mV}$, and that of the C-Type was $680 \mathrm{~Hz}$ with an output voltage of $251 \mathrm{mV}$. These values agree well with the theoretical resonant frequencies of the principal mode.

\section{Conclusions}

A feasibility study of the design technology for miniaturizing an accelerometer was carried out, in which the individual beam has the same length as the side of the proof mass. The sensitivities and the resonant frequencies for three types of MEMS capacitive accelerometer with proof mass sizes of $1000 \times 1000,750 \times 750$, and $500 \times 500 \mu \mathrm{m}^{2}$ were simulated using the FEM simulator "IntelliSuite". Using the design principle of the beam length being as long as that of the side of the proof mass, the sensitivity could be significantly enhanced compared 
with that of the conventional folded-beam accelerometer. In addition, when the proof mass was reduced, the sensitivity could be increased by folding the beams. For the B-Type prototype with $750 \times 750 \mu \mathrm{m}^{2}$ proof mass and a beam folded 3 times, the calculated sensitivity was similar to that of the standard A-Type prototype with $1000 \times 1000 \mu \mathrm{m}^{2}$ proof mass and no beam folding, while the calculated sensitivity for the C-Type prototype with $500 \times 500 \mu \mathrm{m}^{2}$ proof mass and a beam folded 7 times was about half that of the standard prototype. The calculated resonant frequency of the B-Type was similar to that of the A-Type, while the calculated resonant frequency of the C-Type was twice that of the A-Type. The resonant frequencies of higher order modes were larger than the fundamental resonant frequency by more than 4 times for the B-Type and the C-Type. The size of the proof mass and the number of folds in the beams could be decided on the basis of these simulation results. The prototypes of the A-Type, the B-Type and the C-Type were fabricated by SOI-MEMS technology, mounted in DIP28 ceramic packages, and evaluated. The measured results agreed well with the simulated ones. Their downscaling effects on their performance, such as sensitivity and resonant frequency, were investigated, and a folded beam was used to keep the performances unchanged while reducing the proof mass size. Sensors with $750 \times 750 \mu \mathrm{m}^{2}$ proof mass and a 3 -times-folded beam were proposed, where the sensitivity and resonance frequencies were set close to those of the reference A-Type model. For sensors with $500 \times 500 \mu \mathrm{m}^{2}$ proof mass, a 7-times-folded beam was used, but the sensitivity is $1 / 3$ that of the reference Type A whereas the resonant frequency was increased by 2 times. It was revealed that accelerometers with a folded-beam length as long as the side length of the proof mass are quite effective when the proof mass is reduced. By combining a beam length as long as the side length of the proof mass and a folded-beam structure, the device size of the accelerometer could be successfully reduced.

\section{References}

1 A. Bertolini, R. DeSalvo, F. Fidecaro, and A. Takamori: IEEE Trans. Geosci. Remote Sens. 44 (2006) 273.

2 F. A. Levinzon: IEEE/ASME J. Microelectromech. Syst. 12 (2012) 2262.

3 A. Partridge, J. K. Reynolds, B. W. Chui, E. M. Chow, A. M. Fitzgerald, L. Zhang, N. I. Maluf, and T. W. Kenny: IEEE/ASME J. Microelectromech. Syst. 9 (2000) 58.

4 Y. Li, Q. Zheng, Y. Hu, and J. Young: IEEE/ASME J. Microelectromech. Syst. 20 (2011) 83.

5 P. Monajemi and F. Ayazi: IEEE Sens. J. 6 (2006) 39.

6 R. A. Dias, L. Mol, R. F. Wolffenbuttel, E. Cretu, and L. A. Rocha: IEEE Sens. J. 11 (2011) 1677.

7 J. Chae, H. Kulah, and K. Najafi: Proc. 16th IEEE Int. Conf. Micro Electro Mechanical Systems (MEMS) (2003) 466.

8 C. Sun, M. Tsai, Y. Liu, and W. Fang: IEEE Trans. Electron Devices 57 (2010) 1670.

9 K. Ishihara, C. F. Yung, A. A. Ayon, and M. A. Schmidt: J. Microelectromech. Syst. 8 (1999) 403.

10 F. A. Hassania, A. F. Payamb, M. Fathipour, and F. Farbizd: ISDRS 2007 (2007).

11 U. Krishnamoorthy, R. H. Olsso, G. R. Bogart, M. S. Baker, D. W. Carr, T. P. Swiler, and P. J. Clews: Sens. Actuators, A 145 (2008) 283.

12 G. Dai, M. Li, X. He, L. Du, B. Shao, and W. Su: Sens. Actuators, A 172 (2011) 369.

13 E. H. Sarraf, M. Sharma, and E. Cretu: Sens. Actuators, A 186 (2012) 154.

14 S. Hirai, K. Terao, T. Suzuki, F. Shimakawa, and F. Oohira: Proc. 28th Sensor Symp. (2011) 15.

15 J. Liu and H. Muro: IEEJ Trans. Sens. Micromach. 137 (2017) 247. 


\section{About the Authors}

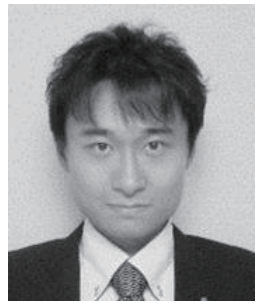

Jiannan Liu received his M.S. degree from Waseda University, Japan. In 2012, he began working at TDK Corporation, Japan. In 2015 he entered the doctoral program course at Chiba Institute of Technology, Japan. Since 2010, his research interests have been MEMS devices and sensors.

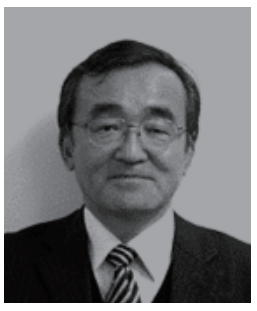

Hideo Muro received his B.S., M.S., and Ph.D. degrees in electronics engineering from the University of Tokyo, Tokyo, Japan, in 1976, 1978, and 1997, respectively. Since joining Nissan Motor Company, Ltd., in 1981, he has been engaged in the research and development of silicon sensors for automotive applications, MEMS devices using bulk micromachining, and sensing technologies for intelligent transport systems (ITS) including optical micro-scanners for laser radar. Since April 2006, he has been a professor in the Department of Electrical, Electronics and Computer Engineering, Chiba Institute of Technology, Chiba, Japan. His current interests are MEMS capacitive accelerometers, silicon resonant sensors, selfsustaining vibration sensors, silicon thermal sensors, optical microscanners, and sensor fusion technologies. He is currently a member of the Institute of Electrical Engineers of Japan, the Society of Automotive Engineers of Japan, and IEEE. 